\title{
A PROPÓSITO DE LA PROPUESTA DE CONTROL DE LOS PLANES DE MANEJO DE ÁREAS PROTEGIDAS DEL ESTADO
}

\author{
Juan C. Monckeberg F. \\ ECOS Chile
}

Kay J. Bergamini L.

P. Universidad Católica de Chile

\author{
José A. Hernández R. \\ Universidad San Sebastián
}

Cristián M. Pérez M.

Universidad de Chile

\begin{abstract}
Resumen: Este artículo identifica problemas en el proyecto de ley que actualmente se tramita en el Congreso relativo al manejo del sistema de áreas protegidas en Chile. Se centra en las atribuciones de gestión, fiscalización y sanción que se espera otorgar al propuesto Servicio de Biodiversidad y Áreas Protegidas respecto del cumpli-
\end{abstract}

Juan C. Monckeberg. Geógrafo de la Pontificia Universidad Católica de Chile (PUC), magíster en derecho ambiental por la Universidad de Chile. Ex superintendente del medio ambiente (SMA). Profesor de magíster en la Universidad de los Andes y Finis Terrae, y de diplomados en el Instituto de Estudios Urbanos y Territoriales, en la PUC. Socio director de consultora ECOS Chile. Email: jcmoncke@uc.cl. José Hernández. Abogado de la Universidad de Chile y magíster (c) en derecho por la misma institución. Ex asesor legal de la SMA. Hoy es asesor legal del Tribunal Ambiental y profesor de derecho ambiental en la Universidad San Sebastián. Email: jhernandezr@docente.uss.cl.

Kay Bergamin. Geógrafo de la PUC y doctor en gestión ambiental, paisaje y geografía por la Universidad de Barcelona. Ex jefe de la división de fiscalización de la SMA. Profesor asistente del Instituto de Estudios Urbanos y Territoriales de la PUC. Socio director de consultora ECOS Chile. Email: kbergani@uc.cl.

CRISTIÁn Pérez. Médico veterinario de la Universidad de Chile. Magíster en gestión y planificación ambiental por la Universidad de Chile. Ex asesor técnico de la SMA. Director del Centro Gestión Ambiental y Biodiversidad de la Universidad de Chile. Socio director de consultora ECOS Chile. Email: cperezm@veterinaria.uchile.cl.

Los autores quieren agradecer los comentarios y la bibliografia aportados por Leonel Sierralta y Andrés Sáez. 
miento de los planes de manejo de áreas protegidas. Concluye que, en lo relativo a las áreas de protección estatales, que cubren el 96 por ciento de las áreas protegidas en el país, el modelo institucional diseñado contiene distorsiones que conllevarán a un cambio apenas simbólico en la materia.

Palabras Clave: medio ambiente, diseño institucional, fiscalización, áreas protegidas, regulation inside government, planes de manejo.

\section{ON THE PROPOSED OVERSIGHT OF MANAGEMENT PLANS FOR STATE PROTECTED AREAS}

ABSTRACT: This article identifies problems with the bill currently before Congress concerning management of the protected areas system in Chile. It centres on the powers of administration, inspection and sanction planned for the proposed Biodiversity and Protected Areas Service in relation to fulfilment of protected area management plans. The conclusion is that in the case of State protected areas, which make up 96 per cent of the country's total, the institutional model designed contains distortions that could well mean that any change is barely more than symbolic.

KeYwords: environment, institutional design, inspection, protected areas, regulation inside government, management plans.

\section{INTRODUCCIÓN}

na reciente publicación ${ }^{1}$ demuestra que la extensa regulación de las áreas protegidas en Chile carece de sistematicidad; que las categorías de protección existentes no son parte de una estrategia de política pública de conservación coherente y armónica; que la gestión de las áreas protegidas carece de una estructura institucional orgánicamente establecida y posee una administración segmentada, efectuada por organismos públicos y privados diversos, con objetivos complementarios de conservación, que debieran estar coordinados; y que la superposición de competencias de diversos organismos públicos respecto de una misma categoría de protección acentúa la difusa responsabilidad del Estado sobre las áreas protegidas.

Con ese contexto, resulta inexplicable que el primer esfuerzo tendiente a establecer un marco legal integrado para las áreas protegidas te-

${ }^{1}$ Sergio Praus et al., La situación jurídica de las actuales áreas protegidas de Chile (Santiago: Andros, 2011), 118. 
rrestres haya quedado sin implementación, por cuanto la Ley 18.384, de 1984 - que establece la Corporación Nacional Forestal y de Protección de Recursos Naturales como una institución autónoma del Estado-, y la Ley 18.362, de 1984 —que establece el Sistema Nacional de Áreas Silvestres Protegidas del Estado, cuyas unidades de manejo administrarían dicha corporación-, permanecen suspendidas indefinidamente condicionadas al decreto de disolución de la Corporación Nacional Forestal (Conaf); agravado por la Ley 18.768, de 1988, que transfirió a esta última la administración de los parques nacionales y reservas forestales, hasta entonces radicada en el Servicio Agrícola y Ganadero (SAG). Incluso, el Tribunal Constitucional, en el control de constitucionalidad de la Ley 20.283, de 2009, sobre recuperación del bosque nativo y fomento forestal, exhortó al ejecutivo a regularizar este problema. ${ }^{2}$

Este llamado fue desoído, a pesar de que entonces se estaba en etapas intermedias de la tramitación de la Ley 20.417, de 2010, retrasando largamente la solución de este problema. A mediados de $2008,{ }^{3}$ la primera administración Bachelet (2006-2010) presentó a trámite legislativo el proyecto de ley para la creación del Ministerio del Medio Ambiente (MMA), el Servicio de Evaluación Ambiental (SEA) y la Superintendencia del Medio Ambiente (SMA), en el que proponía un cambio menor e insuficiente para los desafíos institucionales en materia de áreas protegidas, que apenas consistía en modificar los estatutos de la Conaf, para incorporar al ministro de Medio Ambiente en su consejo directivo, buscando con ello que dicha corporación cumpliese las políticas sobre áreas protegidas definidas por este ministerio. ${ }^{4}$

No obstante, para agilizar la tramitación de la ley, los principales sectores políticos representados en el Senado firmaron un protocolo de acuerdo con la administración Bachelet, destrabando aspectos de diseño organizativo y regulatorio, incluyendo crear el Servicio de Biodiversidad y Áreas Protegidas para reemplazar en esa función a la Conaf. Por tanto, sólo así comenzó una discusión de fondo respecto de la institucionalidad encargada de la protección de la biodiversidad y de las áreas protegidas en Chile.

${ }^{2}$ Control de constitucionalidad del proyecto de ley aprobado por el Congreso Nacional, sobre recuperación del bosque nativo y fomento forestal, Tribunal Constitucional, 1 de julio de 2008, Rol n. ${ }^{\circ}$ 1024-2008.

${ }^{3}$ Mensaje n. ${ }^{\circ} 352-353$, introducido el 5 de junio de 2008.

${ }^{4}$ Biblioteca Congreso Nacional, Historia de la Ley $n .{ }^{\circ}$ 20.417, 22. 
En enero de 2011, la administración Piñera (2010-2014) presentó a trámite legislativo el proyecto de ley para la creación del Servicio de Biodiversidad y Áreas Silvestres Protegidas (SBAP) y el Sistema Nacional de Áreas Silvestres Protegidas (SNAP), ${ }^{5}$ que no tuvo mayor impulso legislativo y fue retirado por la actual administración Bachelet (2014-2018). Ésta, en junio de 2014, presentó a trámite legislativo otro proyecto de ley, el cual con el mismo nombre introdujo cambios respecto del anterior, ${ }^{6}$ pero actualmente está estancado y afronta obstáculos de parte de ministerios del área económica, intereses privados y empleados de la Conaf, así como de ONGs y comunidades indígenas. ${ }^{7}$

Por esto, es necesario analizar si el actual proyecto de ley ofrece un adecuado diseño organizativo e institucional, en particular respecto de la aplicación y fiscalización del Plan de Manejo de Áreas Protegidas (PMAP), principal instrumento de gestión del patrimonio ambiental contenido en el proyecto.

Este trabajo se organiza de la siguiente manera:

La parte 1 introduce una breve explicación conceptual y teórica acerca de instituciones, organizaciones e individuos, para dar paso al enfoque denominado regulation inside government (RIG), impulsado por la OCDE en sus esfuerzos para racionalizar la regulación, mejorar la competitividad, optimizar la asignación presupuestaria del Estado y controlar la calidad de la prestación estatal de servicios públicos, mostrando que para este último objetivo se prefiere un diseño de supervigilancia. Luego se explica como éste se ha aplicado en Chile.

La parte 2 introduce los problemas de gestión de las áreas protegidas en Chile, y explica cuál es el diseño propuesto por el proyecto de ley para los PMAP, particularmente su fiscalización y la sanción de sus infracciones.

La parte 3 expone el actual diseño de la institucionalidad ambiental, en relación con el diseño de control propuesto para los PMAP, desde el enfoque RIG, indicando sus debilidades y mostrando las dificultades que se prevén en su aplicación, a través de su comparación con otro instrumento de gestión ambiental de similares características.

\footnotetext{
${ }^{5}$ Mensaje n. ${ }^{\circ}$ 595-358, introducido el 26 de enero de 2011.

${ }^{6}$ Mensaje n. ${ }^{\circ}$ 161-362, introducido el 18 de junio de 2014.

7 OCDE, Environmental Performance Reviews: Chile 2016 (París: OECD Publishing, 2016), 240.
} 
En la parte final, se concluye que el diseño de control propuesto es insuficiente y que se necesita reformar el diseño de la institucionalidad ambiental, para garantizar realmente la independencia de los reguladores.

\section{INSTITUCIONES, ORGANIZACIONES Y EL ENFOQUE RIG}

\subsection{Individuos, organizaciones e instituciones}

La búsqueda de un adecuado diseño organizativo e institucional es un tema recurrente de política pública, ya que es frecuente que individuos en cargos directivos imputen los fracasos de gestión a errores en el diseño existente, sean reales o no, para victimizarse e intentar evadir su responsabilidad. ${ }^{8}$ Estos diseños organizativos, junto a las reglas formales aplicables a interacciones dentro de la organización, respecto de los componentes de su estructura, y fuera de ella, respecto de otras organizaciones y con los administrados o usuarios, influyen significativamente en la conformación de la institucionalidad. Por tanto, es preciso indagar sobre los conceptos de institución y organización.

Desde una perspectiva sociológica, las instituciones son definidas como patrones de conductas estables, valoradas y recurrentes, ${ }^{9}$ resultantes de entendimientos mutuos de preferencias y de conductas de optimización de individuos y organizaciones, ${ }^{10}$ las que son explicadas por dos aproximaciones: una centrada en reglas y normas, y otra en equilibrios. La primera las concibe como el conjunto de reglas y normas de conducta, formales e informales, y por las características de su cumplimiento, que estructuran la interacción humana, a través de restricciones e incentivos; ${ }^{11}$ mientras que la segunda las concibe como el resultado espontáneo de la conveniencia de los involucrados. ${ }^{12}$

${ }^{8}$ Christopher Hood, The Blame Game: Spin, Bureaucracy, and Self-preservation in Government (Princeton: Princeton University Press, 2011), 30.

9 Samuel Huntington, "Political Development and Political Decay", World Politics 17, n. 3 (1965): 394.

${ }^{10}$ Sue Crawford y Elinor Ostrom, "A Grammar of Institutions", American Political Science Review 89, n. ${ }^{\circ} 3$ (1995): 582.

${ }^{11}$ Douglass North, "Institutions and Economic Growth: A Historical Introduction”, World Development 17 (1989): 1321.

12 Geoffrey Hodgson, "What Are Institutions?", Journal of Economic Issues 40, n. ${ }^{\circ} 1$ (2006): 12. 
En el enfoque centrado en reglas y normas, que estimamos más apropiado y que se adopta en este trabajo, las reglas son prescriptivas, indicando acciones que son requeridas, prohibidas o permitidas; y que resultan de esfuerzos explícitos e implícitos de individuos para obtener orden y predictibilidad en situaciones predefinidas, para lo cual crean cargos, indican cómo se entra y sale de éstos, qué acciones son requeridas, prohibidas o permitidas en su ejercicio, y en qué ámbito aplican. Se advierte que el carácter prescriptivo define a reglas e instituciones, por cuanto significa que los individuos y organizaciones que conocen y aceptan estas reglas reconocen que pueden ser responsabilizados por sus incumplimientos, sea de forma horizontal o vertical, $\mathrm{y}$, en éste último caso, por individuos u organizaciones que específicamente monitorean cumplimiento, resultando imprescindibles los mecanismos de restablecimiento del cumplimiento. ${ }^{13}$

Por tanto, es necesario reconocer las reglas y normas formales e informales de las organizaciones para poder describir y predecir el funcionamiento de las instituciones, y en especial las de las organizaciones emisoras, destinatarias y supervisoras de reglas formales, máxime si integran la estructura del Estado. Éstas últimas se crean y reforman para modificar la institucionalidad, deseablemente de forma eficiente, a partir de oportunidades que surgen desde un conjunto de restricciones con una intención deliberada de modificación de conductas ajenas y propias, y en su accionar para conseguir sus objetivos. En otras palabras, buscan resolver problemas y son un agente de cambio institucional. ${ }^{14}$ Enseguida veremos cómo, en búsqueda de la modificación eficiente de las instituciones, en el sentido sociológico, existe regulación y control estatal dentro de la misma administración.

\subsection{Regulación y control estatal: control de gasto y de calidad}

La regulación y el control son consecuencia del diseño de las organizaciones emisoras, destinatarias y supervisoras de reglas formales. $\mathrm{Si}$ bien existen institucionalidades no estatales que emiten, reciben y

${ }^{13}$ Elinor Ostrom, "An Agenda for the Study of Institutions", Public Choice 48 (1986): 6 .

${ }^{14}$ Douglass North, Institutions, Institutional change, and Economic Performance (Cambridge: Cambridge University Press, 1987), 4. 
supervisan reglas formales, como en el deporte ${ }^{15}$ y en la normalización técnica ${ }^{16}$, nos interesan aquellas que forman parte de la estructura del Estado. No obstante, como se señaló, la efectividad de las reglas depende de mecanismos de control y restablecimiento de su cumplimiento, y como el cumplimiento espontáneo no está garantizado, deben destinarse recursos para prevenir incumplimientos y para detectar y procesar a los incumplidores. ${ }^{17}$

La regulación tradicional es la de actividades de terceros privados, esto es, la que impone el Estado para la protección o el fomento del interés público. ${ }^{18}$ De esta forma, se regula para obtener eficiencia económica y evitar o corregir las denominadas fallas de mercado, como distorsiones por monopolios y monopsonios, abuso de poder de mercado, asimetrías de información y externalidades; pero también para obtener metas sociales, ambientales y culturales, como redistribución del ingreso y riqueza, acceso uniforme a servicios sociales, protección del medio ambiente, asignación de la explotación de recursos naturales escasos, entre otras. ${ }^{19}$

Si bien ha sido mayormente exitosa la privatización total o parcial de empresas estatales dedicadas a la provisión de servicios públicos ligados a monopolios naturales, como servicios sanitarios, distribución eléctrica o telefonía, o dedicadas simplemente a actividades industriales, como producción azucarera, minera y metalúrgica, donde existe mercado nacional e internacional; para la obtención de metas sociales, ambientales y culturales, el mercado suele ser insuficiente, como en la provisión de servicios hospitalarios y educacionales, por cuanto existe exclusión por capacidad de pago del usuario. Incluso existen casos donde para alcanzar metas ambientales no existe naturalmente mercado, pero son creados artificialmente, como ocurre con las regulaciones de comercio de créditos por reducción de emisiones o de asignación de

${ }^{15}$ Graham McFee, Sport, Rules and Values (Londres: Routledge, 2004), 72 y ss.

${ }^{16}$ Nils Brunsson y Bengt Jacobsson, A World of Standards (Oxford: Oxford University Press, 2000), 21 y ss.

${ }^{17}$ Gary Becker, "Crime and Punishment: An Economic Approach", Journal of Political Economy 76, n. ${ }^{\circ} 2$ (1968): 169.

${ }^{18}$ Robert Baldwin et al., Understanding Regulation (Oxford: Oxford University Press, 2012), 4-5.

${ }^{19}$ Ibídem, 24. 
cuotas de extracción de recursos pesqueros; o en su defecto se establecen medidas de fomento de conservación, con pago por servicios ambientales y con rebajas o recargos tributarios, según corresponda.

En el caso de servicios públicos y sociales de provisión mixta, existe regulación que necesariamente se aplica tanto a proveedores privados como públicos. Cuando actúa sobre proveedores privados no suele ser un problema, pero la regulación de proveedores públicos sí. A esta última se aplica el enfoque regulation inside government (RIG), dada la importancia de la eficiencia del gasto público para la provisión de estos servicios y los requerimientos de calidad de su prestación. Este enfoque se manifiesta en la creación o refuerzo de organizaciones estatales que desarrollan regulaciones relacionadas, las vigilan y hacen cumplir por diversos mecanismos; siendo particularmente interesante la regulación de la calidad y efectividad del servicio público o social prestado. ${ }^{20}$

Como el enfoque RIG ha estado ligado a la preocupación por controlar el gasto público, la OCDE considera que la agenda pública de calidad regulatoria debe incluir este enfoque, insistiendo en que la aplicación de principios de calidad regulatoria dentro del sector público y en la interfaz de los sectores público y privado es tan importante como su aplicación al sector privado, debiendo sujetarse el sector público a los mismos estándares de eficiencia, efectividad, transparencia y responsabilidad. ${ }^{21}$

\subsection{El enfoque RIG y la preferencia del diseño de supervigilancia}

El enfoque RIG se desarrolla dentro de las teorías de administración pública de origen británico denominadas New Public Management, o "Nueva administración pública", que combinan lo que se denomina nueva economía de instituciones (new institutional economics) y, por una parte, se basa en teorías de elección pública (public choice), costos de transacción (transaction costs), problema del agente principal (principal-agent) y modelo de maximización presupuestaria (budgetmaximizing model); y, por la otra, en lo que se denomina administración

${ }^{20}$ Ibídem, 6.

${ }^{21}$ OCDE, Taking Stock of Regulatory Reform (París: OECD Publishing, 2005), 15. 
científica (scientific managerialism), pero aplicada a la administración pública. ${ }^{22}$

Este enfoque se ha aplicado en Chile en el denominado proceso de modernización del Estado, ${ }^{23}$ introduciendo reformas parciales de gerencia pública, profesionalizando la función directiva, con un sistema de selección de alta dirección pública, e incentivando la eficiencia, con sistemas de evaluación del desempeño. ${ }^{24}$ Como resultado, se estableció un sistema de evaluación y control de gestión, para mejorar la eficiencia y eficacia del gasto público, vinculando asignación y uso de recursos a resultados de gestión. Éste está compuesto por instrumentos de seguimiento y evaluación — balances de gestión integral, evaluaciones de programas gubernamentales, indicadores de desempeño institucional, formato estándar de presentación de iniciativas al presupuesto- y mecanismos de incentivo al desempeño: programa de mejoramiento de la gestión, metas de eficiencia institucional y convenios de desempeño colectivo. $^{25}$ Pero como se verá enseguida, también existen reformas sectoriales asociadas al control de la calidad del servicio público o social entregado, y su lógica se aproxima mucho más a la de la regulación tradicional.

En todo caso, sea que la agenda reformista recaiga sobre la reestructuración burocrática, entendida como el uso eficiente de recursos humanos y financieros, todos asociados a asuntos presupuestarios y gerenciales, o recaiga en la calidad de la provisión de servicios, ${ }^{26}$ es necesario, como se ha repetido, que existan dos grupos de organizaciones: uno compuesto por aquellas que establecen estándares por medio de regulaciones, los vigilan y hacen cumplir; y otro compuesto por organizaciones que deben cumplir dichos estándares.

${ }^{22}$ Christopher Hood, “A Public Management for all Seasons?”, Public Administration 69 (1991): 5-6.

${ }^{23}$ Verónica Figueroa et al., "Política de modernización de la gestión pública en Chile 1990-2006", Convergencia 18, n. ${ }^{\circ} 57$ (2011): 61-99.

${ }^{24}$ Marjorie Morales, "Nueva gestión pública en Chile: orígenes y efectos", Revista de Ciencia Política 34, n. ${ }^{\circ} 2$ (2014): 420-422.

${ }^{25}$ Rodrigo Castro et al., "Evaluación y propuesta del sistema de control estratégico y operacional de Chile", en Un mejor Estado para Chile, ed. Visnja Tomicic y Cristián García (Santiago: Andros Impresores, 2009), 591.

${ }^{26}$ Martin Lodge y Kai Wegrich, Managing Regulation: Regulatory Analysis, Politics and Policy (Londres: Palgrave Macmillan, 2012), 120-121. 
Por eso, el enfoque RIG considera necesario identificar una organización estatal que tenga como misión legal modelar las actividades de otra organización estatal; si existe algún grado de separación organizacional entre ambas, y excluirse, por tanto, todos los controles y sistemas de cumplimientos internos de la segunda organización. ${ }^{27}$

En este enfoque es posible reconocer sus componentes según funciones, para distinguir entre las organizaciones reguladoras que cumplen funciones de: i) "dirección”, emitiendo estándares; ii) "detección”, recolectando información de su cumplimiento; y iii) "modificación", cambiando las conductas infractoras. ${ }^{28} \mathrm{~A}$ su vez, es posible reconocer diseños regulatorios bajo este enfoque, que pueden presentarse solos o combinados: ${ }^{29}$ i) de supervigilancia, ii) de competencia, iii) de mutualidad y iv) de aleatoriedad planificada, donde el más extendido para el control de calidad de la prestación es el diseño de supervigilancia. ${ }^{30}$

Esta supervigilancia es común en sectores como salud ${ }^{31}$ y educación ${ }^{32}$, y en otros países se ha extendido al sistema de prisiones públicas $^{33}$ y fuerza policial ${ }^{34}$. No obstante, no deja de ser problemático, pues a diferencia de la regulación tradicional, donde el Estado impone medidas coercitivas sobre el administrado para forzar el cumplimento,

${ }^{27}$ Hood et al., Regulation Inside Government: Waste-Watchers, Quality Police, and Sleaze-Busters (Oxford: Oxford University Press, 1999), 8-10.

${ }^{28}$ Ibídem, 45-46.

${ }^{29}$ Martin Lodge y Christopher Hood, "Regulation Inside Government: Retro-Theory Vindicated or Outdated?", en The Oxford Handbook of Regulation, ed. Robert Baldwin, Martin Cave y Martin Lodge (Oxford: Oxford University Press, 2010), 599. También en Lodge y Wegrich, Managing Regulation, 127; y en Hood et al., Regulation Inside Government, 79.

${ }^{30}$ Hood et al., Regulation Inside Government, 48.

31 Daniel Simonet, "The New Public Management Theory and European Health-Care Reforms", Canadian Public Administration 54, n. ${ }^{\circ} 4$ (2008): 617-635.

32 Sowaribi Tolofari, "New Public Management and Education", Policy Futures in Education 3, n. ${ }^{\circ} 1$ (2005): 75-89. En Chile, Alejandra Falabella, "El mercado escolar en Chile y el surgimiento de la nueva gestión pública", Educação \& Sociedade 36, n. ${ }^{\circ} 132$ (2015): 699-722.

${ }^{33}$ Arjen Boin et al., 'The New Public Management 'Revolution' in Political Control of the Public Sector: Promises and Outcomes in Three European Prison Systems", Public Policy and Administration 21, n. ${ }^{\circ} 2$ (2006): 81-100.

${ }^{34}$ Garth den Heyer, "New Public Management: A Strategy for Democratic Police Reform in Transitioning and Developing Countries", Policing: An International Journal of Police Strategies \& Management 34, n. 3 (2011): 419-433. 
tal opción es muy limitada cuando se trata de organizaciones estatales. ${ }^{35}$ Dada la verticalidad de la administración pública y la casi nula distancia organizacional, las relaciones entre organizaciones generalmente se caracterizan por interacciones cooperativas, un interés común por evitar la vigilancia y las críticas externas, y el deseo de mantener relaciones de control informal. ${ }^{36}$

Entonces, ¿cómo hemos aplicado este enfoque en algunos sectores en Chile?

\subsection{El enfoque RIG aplicado en Chile para el control de calidad del servicio}

La preocupación por la cobertura y la calidad del servicio público o social ha motivado en Chile la introducción del enfoque RIG en los sistemas de provisión de salud y educación. En ambos casos existe provisión mixta $y$, en consecuencia, las consideraciones regulatorias deben ser uniformes. Sin embargo, se han observado severos problemas de coercibilidad de estándares en la provisión pública, coincidente con las limitaciones inherentes del control del Estado por sí mismo, ya señaladas anteriormente. Explicar brevemente estos sistemas permite comprender mejor cómo es posible aplicarlos en materias ambientales, aun cuando parezca que en éste ámbito no existe una prestación de servicio público en un sentido clásico.

En el control de calidad en la provisión mixta de salud, con la reforma introducida por la Ley 19.937, de 2004, es posible diferenciar entre funciones de "dirección", "detección” y "modificación" que desempeñan organizaciones reguladoras sobre organizaciones reguladas, bajo un modelo de supervigilancia. ${ }^{37} \mathrm{La}$ "dirección" es del Ministerio de Salud, que establece estándares específicos de calidad de servicio, respecto de los que deben acreditarse los prestadores institucionales públicos y privados. La "detección” es de la Superintendencia de Salud, que identifica desviaciones a dichos estándares, administrando un mecanismo de acreditación de prestadores institucionales con el objetivo

\footnotetext{
${ }^{35}$ Lodge y Wegrich, Managing Regulation, 123.

${ }^{36}$ Lodge y Hood, "Regulation Inside Government", 594-595.

${ }^{37}$ Paula Benavides et al., Sistema público de salud: Situación actual y proyecciones fiscales 2013-2050, (Santiago: Dipres, 2013), 9-20.
} 
de garantizar que sus prestaciones alcancen la calidad requerida para la seguridad de los usuarios, y además los fiscaliza en la mantención del cumplimiento de los estándares de acreditación. La "modificación" es del Ministerio de Salud y la Superintendencia de Salud, por diversas vías, pero esta última lo hace aplicando sanciones a prestadores institucionales, aunque tratándose de prestadores públicos en su gran mayoría se los sanciona con amonestación y, marginalmente, con multa. ${ }^{38}$

En el control de calidad en la provisión mixta de educación, con la reforma introducida por la Ley 20.529, de 2011, también es posible la diferenciación de funciones bajo un modelo de supervigilancia. $\mathrm{La}$ "dirección" es del Ministerio de Educación, que desarrolla un Plan de Aseguramiento de la Calidad de la Educación, quedando los establecimientos educacionales de enseñanza parvularia, básica y media, sean públicos municipales, particulares subvencionados o particulares pagados, obligados a cumplirlo. La "detección” es tanto de la Superintendencia de Educación como de la Agencia de Calidad de la Educación: la primera fiscaliza el cumplimiento de los requisitos exigidos para mantener el reconocimiento oficial del Estado, y la segunda es responsable de evaluar el desempeño de estos establecimientos basándose en estándares indicativos, e informa periódicamente a la Superintendencia de Educación para la imposición de sanciones en caso de incumplimiento de estos estándares nacionales de aprendizaje. La "modificación" es del Ministerio de Educación y la Superintendencia de Educación, por diversas vías, pero esta última lo hace aplicando sanciones a establecimientos públicos municipales, particulares subvencionados y privados, aunque tratándose de establecimientos públicos municipales mayormente se los sanciona con amonestación y, marginalmente, con multa. ${ }^{39}$

${ }^{38}$ Como la Res. Ex. IP n. ${ }^{\circ}$ 0303/2014, que aplica sanción de 50 UTM al Hospital Base de Linares, perteneciente al Servicio de Salud Maule; o la Res. Ex. IP n. ${ }^{\circ}$ 0620/2015, que aplica sanción de 60 UTM al Hospital Dr. Eduardo Pereira, perteneciente al Servicio de Salud Valparaíso - San Antonio. En todo caso, la sanción habitual a los hospitales públicos es la amonestación por escrito.

${ }^{39}$ Como la Res. Ex. n. ${ }^{\circ}$ 2013/PA/01/121, que aplica sanción de 69 UTM al establecimiento educacional Escuela Básica Chipana, RBD n. ${ }^{\circ} 12538-5$, cuyo sostenedor es la Corporación Municipal de Desarrollo Social de Iquique; o la Res. Ex. n. ${ }^{\circ}$ 2012/PA/05/0197, que aplica sanción de revocación del reconocimiento oficial del Estado al establecimiento educacional Escuela Básica Japón, RBD n. ${ }^{\circ}$ 1545-8, cuyo sostenedor es la Corporación Municipal de Valparaíso para el Desarrollo Social. 
Nótese que el reciente proyecto de ley del Sistema de Educación Pública introducido por la actual administración Bachelet (2014-2018) en noviembre de $2015,{ }^{40}$ por el cual se traspasará los establecimientos municipales a los propuestos Servicios Locales de Educación Pública, deja intacta las atribuciones de la Superintendencia de Educación en materia de control de calidad. Pero acá se propone una opción novedosa, pues su artículo 16 dispone la cesación del cargo de director ejecutivo del Servicio Local de Educación por negligencia manifiesta en el desempeño de sus funciones, entendiendo como tal cuando el Servicio Local de Educación Pública o los establecimientos bajo su dependencia incurren en reiteración de infracciones graves impuestas por la Superintendencia de Educación.

Incluso en los servicios de previsión social, en el ámbito de pensiones, puede reconocerse un enfoque RIG. Con la reforma introducida por la Ley 20.225, de 2008, el Instituto de Previsión Social administra el sistema solidario, en especial, la concesión, extinción, suspensión o modificación de sus beneficios, y, además, administra los regímenes de prestaciones de las extintas cajas de previsión y Servicio de Seguro Social. Sin embargo, la Superintendencia de Pensiones supervigila y fiscaliza el sistema y los regímenes antes señalados, y sanciona el incumplimiento de las normas aplicables a estos, así como los aplicables a las administradoras de fondos de pensiones. Tal como sucede en educación y salud, las sanciones pecuniarias que ha sufrido el Instituto de Previsión Social han sido marginales. ${ }^{41}$

Pero en otros casos el enfoque RIG basado en la supervisión parece en retirada. El reciente proyecto de ley de educación superior introducido por la actual administración Bachelet (2014-2018), en julio de 2016, ${ }^{42}$ propone un Sistema Nacional de Aseguramiento de la Calidad, basado también en la diferenciación de las funciones de "dirección", "detección” y "modificación". Sin embargo, a pesar de proponer una Superintendencia de Educación Superior, su fiscalización se centra casi únicamente en los controles financieros de las instituciones de educa-

${ }^{40}$ Mensaje n. ${ }^{\circ} 1.174-363$, introducido el 2 de noviembre de 2015.

${ }^{41}$ Como la Res. Ex. n. ${ }^{\circ}$ 64/2013, que aplica sanción de 300 UF; o la Res. Ex. n. ${ }^{\circ}$ 10/2013 que aplica sanción de 600 UF, en ambos casos al Instituto de Previsión Social.

${ }^{42}$ Mensaje n. ${ }^{\circ}$ 110-364, introducido el 6 de abril de 2016. 
ción superior, pero con sanciones aplicables a aquellas que se organicen como personas jurídicas de derecho privado sin fines de lucro, mientras que las instituciones de educación superior del Estado sólo serán fiscalizadas por la Contraloría General.

En resumen, el enfoque RIG se ha aplicado extensivamente en Chile, en especial respecto de la eficiencia del gasto público, de los mecanismos de ingreso a cargos directivos y de los incentivos de eficiencia en la gestión; y, en menor medida, respecto del control de calidad del servicio prestado.

\section{EL DISEÑO PROPUESTO PARA EL CONTROL DE LOS PMAP}

\subsection{El déficit de gestión de las áreas protegidas}

La administración Lagos (2000-2006) elaboró la Política Nacional de Áreas Protegidas en 2005, en la que se subrayaba la necesidad de estructurar un sistema nacional para estas áreas, con las adecuaciones normativas e institucionales necesarias para optimizar su gestión y asegurar su protección efectiva y eficiente, bajo un modelo de gestión que consideraba liderazgo, planificación, administración, regulación, fiscalización y seguimiento, comprometiendo los recursos públicos necesarios. $^{43}$

Pero todo quedó en el papel. La gestión de las áreas protegidas en Chile es deficiente, percepción que es extendida entre los individuos que conforman las organizaciones públicas relacionadas con dicha tarea. Un estudio por medio de encuestas aplicadas a éstos demostró que consideraban que la gestión era sumamente deficitaria, en particular respecto de problemas de suficiencia de personal, presupuesto, capacidad de fiscalización e implementación de los planes de manejo de áreas protegidas (PMAP), todo esto a pesar de las presiones a que fueron sometidos por sus superiores para entregar respuestas menos pesimistas. ${ }^{44}$ De acuerdo a este estudio, la falta de recursos para mejorar la gestión es 11.

${ }^{43}$ Conama, Política Nacional de Áreas Protegidas (Santiago: Conama, 2005),

${ }^{44}$ Eduardo Fuentes y Rodolfo Domínguez, Aplicación y resultados de la Encuesta sobre Efectividad de Manejo de las principales áreas protegidas de Chile (Santiago: Andros, 2011), 101-105. 
una queja uniforme y transversal, pero también destaca un marco legal insuficiente, la falta de personal permanente y las severas restricciones presupuestarias, con la consecuencia de que los PMAP no pueden implementarse plenamente, sino sólo las acciones que permitan el presupuesto disponible, a través de planes operativos anuales. ${ }^{45}$

El diseño, ejecución y control de cada PMAP es fundamental para el éxito del objetivo de conservación de toda área protegida, al punto de que un estudio ${ }^{46}$ para proponer un sistema integral de áreas protegidas en Chile identifica riesgos administrativos y financieros que afectan la elaboración e implementación de los PMAP, no sólo por la falta de personal calificado para tales tareas, sino también por el excesivo destino de personal a labores administrativas, advirtiendo que diseñar estos planes será inútil si no se cuenta con el personal necesario para implementarlos, en cantidad y especialización.

También es importante señalar que, según la reciente evaluación de desempeño ambiental de Chile, nuestro país enfrenta desafíos considerables en la gestión de sus áreas protegidas. A pesar de que el 80 por ciento de las áreas protegidas terrestres tienen un PMAP, estos se ejecutan parcialmente, están incompletos o necesitan ser revisados y actualizados. Además, la mayoría de las áreas protegidas carecen de suficientes recursos financieros y humanos, ${ }^{47}$ y se advierte que, según la versión preliminar del Plan de Acción de Áreas Protegidas para 20152030, es probable que Chile no cuente con PMAP plenamente operacionales en todas sus áreas protegidas hasta el $2050 .{ }^{48}$ En ese contexto, ¿qué propone el proyecto respecto del principal instrumento de gestión de la biodiversidad?

\subsection{El control propuesto para los PMAP}

Como se señaló, el futuro Servicio de Biodiversidad y Áreas Silvestres Protegidas (SBAP) tendrá a su cargo la administración del Sistema Nacional de Áreas Silvestres Protegidas (SNAP), que estará conforma-

\footnotetext{
${ }^{45}$ Ibídem, 111-113.

${ }^{46}$ Guillermo Espinoza, Evaluación ambiental estratégica de apoyo al diseño del Sistema Nacional de Áreas Protegidas (Santiago: Andros, 2011), 193.

${ }^{47}$ OCDE, Reviews of Regulatory Reform: Regulatory Policy in Chile (Paris: OECD Publishing, 2016), 250.

${ }^{48}$ Ibídem, 251.
} 
do por las áreas protegidas del Estado (APE) — creadas en espacios de propiedad fiscal o en bienes nacionales de uso público, incluyendo la zona económica exclusiva - y por áreas protegidas de propiedad privada (APP), creadas en espacios de propiedad privada y reconocidas conforme a las disposiciones de la ley propuesta. Estas áreas, privadas o estatales, se clasificarán en algunas de las siguientes categorías: reserva de región virgen, parque marino, parque nacional, monumento natural, reserva marina, reserva nacional, santuario de la naturaleza, área marina y costera protegida de múltiples usos y humedal de importancia internacional. ${ }^{49}$

De esta manera, el proyecto de ley concibe que el SBAP administre las áreas protegidas estatales y supervise la administración de las privadas, así como elabore los PMAP de las primeras y apruebe los de las segundas, y vele por su cumplimiento en ambas modalidades. ${ }^{50}$

Respecto a la administración de las áreas protegidas estatales, el Cuerpo de Guardaparques del SBAP será la autoridad competente para su manejo y fiscalización, debiendo velar por el mantenimiento de su equipamiento, infraestructura y bienes, ejerciendo funciones de control y fiscalización en el cumplimiento del PMAP, y de las obligaciones de concesionarios y titulares de permisos que operen dentro del área, entre otras funciones. Además, el SBAP nombrará un administrador del área protegida estatal, seleccionado dentro del Cuerpo de Guardaparques, que será responsable de velar por la aplicación y el cumplimiento del PMAP, entre otras funciones. ${ }^{51}$ Por otra parte, la administración de las áreas protegidas privadas será realizada por sus propietarios o por organizaciones sin fines de lucro, pero el SBAP supervisará dicha gestión y manejo, lo que incluye aprobar sus PMAP. ${ }^{52}$

El proyecto de ley concibe, para la adecuada conservación y administración de las áreas protegidas, que éstas deban obligatoriamente contar con un PMAP consistente con sus objetivos de gestión de la biodiversidad, que además deberá actualizarse cada cinco años. De tal forma, el PMAP corresponde a un instrumento de gestión que establece los aspectos técnicos, normativos y las acciones que se requieren para garantizar la conservación del objeto de un área protegida, establecien-

\footnotetext{
${ }^{49}$ Artículo 2, letra b) y c), y artículos 14 a 22.

${ }^{50}$ Artículo 6.

${ }^{51}$ Artículos 35 a 37 del proyecto de ley.

${ }^{52}$ Artículo 59 a 61.
} 
do una zonificación, objetivos y programas que definan los usos y prohibiciones dentro del área protegida. ${ }^{53}$

Además, el diseño del PMAP tiene que cumplir con ciertas características: i) debe incluir metas medibles y un período para implementarlas, para evaluar su éxito y adecuar recursos y capacidades si fuese necesario; ii) debe señalar de manera precisa aquellas prohibiciones y regulaciones de determinadas actividades en el área protegida o en zonas específicas de la misma, en conformidad a la legislación; y iii) debe establecer los objetivos específicos, las medidas de manejo y los recursos necesarios para su implementación, pudiendo contener programas o planes de acción específicos para conservación, uso sostenible, investigación científica, monitoreo, educación, recreación, ecoturismo, aspectos regulatorios, administración y coordinación. ${ }^{54}$ También debe cumplir con requisitos de contenido: debe informar los antecedentes jurídicos del área; la descripción general del área y sus atributos y valores de conservación; el funcionario encargado de su manejo y otros aspectos de su gobernanza; las principales amenazas y las estrategias de manejo para mitigarlas o suprimirlas; la zonificación; las actividades compatibles; el plan de monitoreo y seguimiento; y los indicadores para evaluar el avance de las metas y objetivos, y la eficacia de diversos enfoques específicos de manejo. ${ }^{55}$

Si bien, los PMAP deberán revisarse cada cinco años, en el caso de la creación de nuevas áreas protegidas, el plazo para dictarlo será de dos años desde la creación de ésta, siempre que exista disponibilidad presupuestaria para ejecutarlo; no obstante, un reglamento establecerá el procedimiento administrativo para la elaboración de los PMAP, así como los contenidos específicos según categoría. ${ }^{56}$

\subsection{Fiscalización, infracciones en áreas protegidas y sanciones}

El proyecto de ley señala que corresponderá al SBAP fiscalizar el cumplimiento de los PMAP de las áreas protegidas estatales, así como de las obligaciones establecidas en los contratos de concesión, de los

\footnotetext{
${ }^{53}$ Artículo 3, letra p).

${ }^{54}$ Artículo 30.

${ }^{55}$ Artículo 31.

${ }^{56}$ Artículos 33 y 34.
} 
permisos otorgados en las áreas estatales y privadas, y en general de todas las actividades que allí se desarrollen. ${ }^{57}$ Pero en el catálogo de infracciones se tipifica genéricamente como tales a toda acción u omisión contraria al PMAP, cometida dentro de cualquier área protegida, para inmediatamente establecer una serie de tipos infraccionales específicos, donde llama la atención el constituido por el incumplimiento de alguna disposición del PMAP por parte del titular de un área, su administrador o el concesionario. ${ }^{58}$ Sobre este aspecto se volverá más adelante.

En cuanto a las categorías de las infracciones, el proyecto de ley considera gravísima aquella que haya causado daño ambiental irreparable, que haya afectado gravemente los servicios ecosistémicos o que haya impedido u obstaculizado deliberadamente el cumplimiento del PMAP. Luego considera grave aquella que haya causado daño ambiental reparable, que haya afectado el área protegida sin comprometer gravemente los servicios ecosistémicos que ésta provee o que haya afectado negativamente el cumplimiento del PMAP. Por último, considera leve, aquella que no sea gravísima ni grave. ${ }^{59}$

En lo relativo a las sanciones, el proyecto de ley señala que en caso de infracciones gravísimas se podrá imponer hasta 10.000 UTM de multa, la desafectación definitiva del área protegida privada, la revocación de la concesión o permiso según corresponda, y de cinco a diez años de prohibición temporal de ingreso a áreas protegidas. En caso de infracciones graves, se podrá imponer hasta 5.000 UTM de multa, la desafectación temporal de la APP, la suspensión de la concesión o permiso según corresponda, y de uno a dos años de prohibición temporal de ingreso a áreas protegidas. Y en caso de infracciones leves, se podrá imponer hasta 500 UTM de multa. Se indica que el fisco deberá traspasar todas las multas recaudadas al presupuesto del SBAP. ${ }^{60}$

En relación al procedimiento administrativo sancionatorio, grosso modo, el proyecto de ley señala que éste podrá tener como antecedente el acta de fiscalización del guardaparques, enviada al director regional del SBAP, o la denuncia de cualquier persona, si es admitida tras un análisis de seriedad y mérito; luego el director regional designará un

\footnotetext{
${ }^{57}$ Artículo 86.

${ }^{58}$ Artículo 92, letra t).

${ }^{59}$ Artículo 94.

${ }^{60}$ Artículo 96.
} 
instructor, quien emitirá una resolución que contendrá una formulación precisa de los cargos, $\mathrm{y}$, si es el caso, se pronunciará sobre las medidas provisionales; una vez notificada dicha formulación de cargos, se concibe un plazo para oponerse, y se indica que el instructor, tras transcurrir este plazo y según el mérito de los antecedentes, podrá abrir una etapa probatoria, en la que los hechos investigados podrán acreditarse por cualquier medio de prueba admisible en derecho, y que la prueba se apreciará según la sana crítica. Por último, el instructor evacuará un informe, que deberá contener, entre otros aspectos, la relación de los hechos investigados y la forma cómo se han comprobado, y la proposición de sanción o absolución de uno o más infractores, remitiendo los antecedentes al director regional para que éste resuelva. ${ }^{61}$

En cuanto a los recursos contra la resolución sancionatoria, se propone el agotamiento previo de la vía administrativa para poder acceder a la tutela judicial. Procede el recurso administrativo jerárquico ante el director nacional del SBAP, y contra la resolución de éste procede el recurso jurisdiccional de reclamación ante el Tribunal Ambiental competente. ${ }^{62}$

Finalmente, una vez notificada la resolución sancionatoria, el proyecto de ley establece la posibilidad de que el infractor presente ante el SBAP una propuesta de plan de reparación de la pérdida o degradación causada en la biodiversidad. ${ }^{63}$ Esta propuesta de plan de reparación se presentará ante el director nacional del SBAP, quien deberá emitir un informe de la infracción cometida y los efectos ocasionados (lo que ya debiese estar contenido en la resolución sancionatoria) y remitirlo junto con el plan propuesto al Ministerio del Medio Ambiente, el cual procederá a su aprobación o eventual rechazo. Su aprobación suspenderá el plazo de prescripción de la acción por daño ambiental, y si se ejecutare satisfactoriamente la acción se extinguirá. Pero en caso de no presentar el plan de reparación, o de no ejecutarse satisfactoriamente, el Consejo de Defensa del Estado deberá ejercer la acción por daño ambiental ante el Tribunal Ambiental competente.

\footnotetext{
${ }^{61}$ Artículos del 97 al 106.

${ }^{62}$ Artículos 107 y 108.

${ }^{63}$ Artículo 110.
} 


\section{LA PROPUESTA DE CONTROL DE LOS PMAP SEGÚN EL ENFOQUE RIG}

\subsection{El actual diseño de la institucionalidad ambiental}

Una aproximación rápida a la interrelación funcional introducida por la Ley 20.417, de 2010, que reestructura el diseño organizativo de la administración en asuntos ambientales, permite reconocer algunos aspectos del enfoque regulatorio tradicional, y también del enfoque RIG.

En ese sentido, el MMA es concebido como el órgano superior de colaboración del Presidente en el diseño y aplicación de políticas, planes y programas en materia ambiental, así como en la protección y conservación de la diversidad biológica y de los recursos naturales renovables e hídricos, promoviendo el desarrollo sustentable, la integridad de la política ambiental y su regulación normativa. A través suyo, se tramitan los procedimientos administrativos para la dictación de normas de calidad, normas de emisión y planes de prevención y/o de descontaminación; se interpretan administrativamente estas regulaciones, y puede exigir y aprobar la presentación de planes de manejo de recursos renovables, denominados planes de manejo de la Ley 19.300, de 1994, que se diferencian de otros planes de manejo de recursos naturales renovables exigidos por otras leyes.

Por su parte, el SEA es concebido como un servicio público funcionalmente descentralizado, con personalidad jurídica y patrimonio propio, sometido a la supervigilancia del Presidente a través del MMA, y está encargado de administrar el Sistema de Evaluación de Impacto Ambiental (SEIA), y de coordinar a todos los organismos sectoriales con competencias en evaluación ambiental, entregando la opinión técnica necesaria para la calificación ambiental y eventual autorización de los proyectos.

A su turno, la SMA también es concebida como un servicio público funcionalmente descentralizado, con personalidad jurídica y patrimonio propio, sometido a la supervigilancia del Presidente a través del mismo ministerio, y está encargado de ejecutar y coordinar la fiscalización de las normas de calidad, normas de emisión, planes de prevención y/o de descontaminación, en lo que corresponda, así como de las resoluciones de calificación ambiental y de los planes de manejo de recursos natura- 
les renovables de la Ley 19.300, de 1994, y sancionar administrativamente sus eventuales incumplimientos.

Esta referencia a los planes de manejo de recursos naturales renovables es interesante. Respecto de ellas se ha señalado que su intención es complementar la vocación productiva de los planes de manejo existentes en otras leyes, con consideraciones ambientales, recordando que la obligación de asegurar la capacidad de regeneración del recurso no es una obligación que pueda ser impuesta caso a caso, sino manejada integralmente, como en parques nacionales ${ }^{64}$. Por tanto, si bien los PMAP no parecen estar comprendidos dentro de esta clase de planes de manejo, pueden ser asimilados, por cuanto su finalidad es asegurar la conservación de los recursos naturales que son usados o aprovechados en un área determinada. No obstante, la diferencia radica en que la elaboración e implementación de los planes de manejo de recursos naturales renovables son impuestas discrecionalmente a los administrados, mientras que los PMAP constituyen un deber de la administración.

En ese sentido, el proyecto de ley propone modificar el artículo 42 de la Ley 19.300, de 1994, sustrayendo expresamente a los PMAP de los planes de manejo de recursos naturales renovables allí contenidos, $\mathrm{y}$, en consecuencia, excluye definitivamente también la competencia fiscalizadora y sancionadora de la SMA respecto de éstos. ${ }^{65}$ Esta modificación, que pudiese parecer muy menor, es muy relevante, como se verá enseguida.

\subsection{Los problemas del diseño de control propuesto para los PMAP}

Como se señaló, el proyecto de ley concibe que el SBAP estará a cargo del diseño y ejecución de los PMAP, así como de su fiscalización, $\mathrm{y}$, consecuentemente, de la imposición de sanciones administrativas por infracción a éstos. Así, el mensaje de la ley afirma que la eficacia de la propuesta depende en buena medida del régimen de fiscalización y sanción que se establezca $y$, por tanto, se propone dotar al SBAP de facultades para fiscalizar los instrumentos de conservación. ${ }^{66}$ Pero

${ }^{64}$ Gabriel del Fávero, "Ley sobre bases generales del medio ambiente", Estudios Públicos 54 (1994): 26-27.

${ }^{65}$ Artículo 113 del proyecto de ley.

${ }^{66}$ Mensaje n. ${ }^{\circ} 161-362,24$. 
también se ha señalado que, en el catálogo de infracciones contenido en el proyecto, se tipifica genéricamente como tales a toda acción $\mathrm{u}$ omisión contraria al PMAP, cometida dentro del área protegida, con independencia de si ésta es estatal o privada, y especificándose que existe infracción por el incumplimiento de alguna de las disposiciones del PMAP por parte del titular de un área protegida, su administrador o el concesionario, sea éste público o privado.

En ese sentido, la racionalidad detrás de un PMAP no es muy distinta a la de los planes de prevención y descontaminación atmosférica (PPDA), pues ambos establecen obligaciones para los regulados y también para la administración. Aunque parezca extraño, las obligaciones para la administración de un PPDA van desde acciones materiales, como el dictar talleres o el recambio de calefactores, hasta acciones formales o normativas, como determinar medidas de restricción vehicular o autorizar compensaciones de emisiones, de las cuales depende el cumplimiento de las metas establecidas. El control de la actividad administrativa de implementación de los planes de prevención o de descontaminación fue un objeto tangencial de debate durante la tramitación de la reforma ambiental, donde se afirmó que la Superintendencia del Medio Ambiente (SMA) tendría potestades para hacer que la administración cumpliera las obligaciones impuestas en dicha normativa, llenando así un vacío regulatorio asociado a la inexistencia de supervisión especializada del cumplimiento de obligaciones propias de la administración. ${ }^{67}$

Precisamente reconociendo los problemas políticos de aplicar tal posición, la SMA dictó una instrucción general sobre registro y reporte del estado de avance de estos planes, ${ }^{68}$ en la que distingue las medidas de control regulatorio, respecto de los administrados, y las medidas de implementación, siendo estas últimas aquéllas por las cuales los servicios públicos llevan a cabo las medidas asignadas que no sean de control regulatorio. Sin embargo, en dicha resolución la SMA reconoce expresamente que solamente realizará un análisis del cumplimiento del plazo de las medidas, y con los indicadores y medios de verificación y registros efectuará una evaluación cuantitativa o formal del estado de avance. Dejó así a un lado la evaluación de fondo que técnicamente es

\footnotetext{
${ }^{67}$ Biblioteca Congreso Nacional, Historia de la Ley $n .^{\circ} 20.417,867$.

${ }^{68}$ Res. Ex. n. ${ }^{\circ}$ 913/2016.
} 
la relevante y, desde luego, descartó cualquier posibilidad de sanciones. Pero no debe ignorarse que la Contraloría General de la República ha auditado el cumplimiento del PPDA de la Región Metropolitana, ${ }^{69}$ aunque también de forma similar al enfoque de la SMA.

Como se puede deducir, si el nuevo SBAP adoptara esta posición debilitaría el control de las obligaciones de los PMAP estatales.

Sin embargo, como se comentó, Chile ha reformado su institucionalidad en materia de salud y educación, con énfasis en el control de la calidad de la prestación de estos servicios. No existe ninguna dificultad conceptual para enmarcar la conservación de la biodiversidad y la protección del medio ambiente en un sistema similar: la ejecución de un PMAP en un área protegida estatal es una actividad prestacional que desarrollará el SBAP, y que se debe a la comunidad toda, en cuanto el artículo 19 número 8 de la Constitución reconoce el derecho a vivir en un medio ambiente libre de contaminación, con su contrapartida expresa, que es el deber del Estado de velar para que este derecho no sea afectado y tutelar la preservación de la naturaleza. Por tanto, la administración misma del área protegida estatal y la implementación del PMAP — en particular, los planes de monitoreo y seguimiento que éstos obligatoriamente deben incluir - constituyen una actividad prestacional. $\mathrm{Su}$ diseño adecuado debe comprender necesariamente estándares de calidad medibles, y el PMAP debe, por lo tanto, incluir indicadores para evaluar el avance de los objetivos y metas de gestión de la biodiversidad, y la eficacia de enfoques específicos de manejo que permitan conservar e incrementar el patrimonio ambiental de país. Pero tan importante como estos estándares son sus controles, es decir, los mecanismos que permitan observar y evaluar su cumplimiento, restablecerlo cuando éste falle o sancionar su incumplimiento. Esto es de sentido común, ya que, como se ha reconocido, las funciones de cualquier organización son el planteamiento, la ejecución y el control, y en el sector público existe una tendencia a reforzar decrecientemente estas fases. Así, los sistemas de control son mucho menos desarrollados que los de planeamiento. ${ }^{70}$

Curiosamente, el proyecto de ley hoy en discusión propone un control ilusorio sobre los planes de manejo de áreas protegidas. Ello queda

\footnotetext{
${ }^{69}$ Informe final n. ${ }^{\circ} 181$ de 2012, y su informe de seguimiento de 2014.

${ }^{70}$ Gerhard Speidel, "Problemas de administración forestal en América Latina", Revista Bosque 1, n. ${ }^{\circ} 1$ (1975): 47.
} 
de manifiesto por una contradicción básica que se explica a continuación. Toda área protegida debe tener un propietario, un administrador y un PMAP (y eventualmente concesionarios), en tanto el SBAP debe fiscalizar el cumplimiento de este plan (Art. 86 del proyecto de ley) y sancionar su incumplimiento cuando sea cometido por el propietario, el administrador o el concesionario (Art. 92), sin importar si se trata de un PMAP aplicable a un área protegida estatal o un área protegida privada. Por tanto, y he aquí el absurdo de que, cuando se trate de un PMAP de un área protegida estatal, existiría la curiosa situación de que el SBAP debería fiscalizar y eventualmente sancionar al fisco —en su calidad de titular del área protegida estatal — o a sus propios funcionarios - en su calidad de administradores del área protegida estatal—, lo que es jurídicamente dudoso y políticamente imposible.

Esa consideración de diseño hará incurrir probablemente al SBAP en un trato diferenciado entre titulares y administradores de áreas protegidas estatales y privadas, donde los privados llevarían la peor parte en cuanto a sanciones, al comportarse como un sistema de regulación tradicional, mientras que los públicos quedarían permanente impunes por incumplimiento y violaciones idénticas. De esta forma, el único control que quedaría para los PMAP de las áreas protegidas estatales sería aquel que resulta de la Contraloría General de la República, de carácter jurídico, y del Ministerio del Medio Ambiente, de carácter político y técnico. A este mismo resultado puede llegarse si se afirmase que el artículo 92 aplica únicamente a las áreas protegidas privadas, a pesar de ir contra su tenor literal.

Por otro lado, podría afirmarse que la fiscalización y sanción del incumplimiento de los PMAP en las áreas protegidas estatales se refieren a las obligaciones que en razón de ellos tienen los privados, por ejemplo, derivados de violar la zonificación o desarrollar actividades incompatibles; pero esto dejaría en segundo plano las obligaciones del SBAP respecto del plan de monitoreo y seguimiento que dicho instrumento considera y que son el elemento central para hacer gestión de la biodiversidad en dichas áreas. Sin esta información no es posible evaluar si los objetivos del área protegida se cumplen y mucho menos efectuar adecuadamente la revisión quinquenal del PMAP al que obligaría el artículo 33 del proyecto. 
Sin embargo, el proyecto de ley pudo escoger un control menos ilusorio, otorgando a la Superintendencia del Medio Ambiente (SMA) la potestad para fiscalizar y sancionar los incumplimientos del PMAP por parte de sus titulares o administradores, sin importar si se trata de área estatal o privada. En tanto, para las infracciones al PMAP cometidas por los concesionarios, así como para el resto de todas las demás infracciones, mantenerlas en el ámbito de la competencia del Servicio de Biodiversidad y Áreas Silvestres Protegidas (SBAP).

De haberse adoptado dicho esquema, esta forma de control sería muy similar al enfoque RIG aplicado en materias de salud y de educación, lo que además es consistente con el modelo concebido por la Ley 20.417, de 2010, que el actual proyecto modifica, bajo el pobre argumento de que tal decisión, concebida en el enfoque de la regulación tradicional, responde a la especificidad de la materia y a la eficacia de que sean los mismos guardaparques quienes puedan fiscalizar y dar fe de incumplimientos que constaten en terreno. ${ }^{71}$

Sin embargo, reiteramos que no es razonable ni sensato que los guardaparques fiscalicen al administrador de un área protegida estatal respecto del cumplimiento del PMAP, pues el administrador es uno de ellos, además de ser su superior. Desde luego, es razonable y sensato que se considere que todas las infracciones contenidas en el artículo 92, excepto su letra t), que se constaten por guardaparques sean sancionadas por el SBAP, dado que éstas se relacionan con la administración cotidiana del área protegida, los obligados son administrados y terceros, y porque el procedimiento sancionatorio propuesto es más simple que el de la Superintendencia y con multas sustancialmente inferiores.

\section{CONCLUSIONES}

El proyecto de ley contiene una inmensa brecha en materia de fiscalización de los PMAP que se debe remediar. Para corregirla se propone que la fiscalización de éstos se mantenga en la Superintendencia del Medio Ambiente (SMA), conservando mayormente la integridad del modelo elegido por la Ley 20.417, de 2010. Lo anterior es muy relevante, dado que existen 232 sitios de conservación como áreas protegidas

${ }^{71}$ Mensaje n. ${ }^{\circ}$ 161-362, 24. 
estatales, que totalizan 30.209.408 hectáreas, y 157 como áreas protegidas privadas, que totalizan 1.255.341 hectáreas. Por lo tanto, el 96 por ciento de la superficie protegida es estatal, ${ }^{72}$ y toda ella quedaría bajo un modelo de fiscalización que estimamos deficiente y contradictorio con el modelo que se propuso en la reforma ambiental del año 2010, que buscaba la separación de funciones.

Adoptando un esquema bajo el enfoque RIG se permitiría controlar de manera más eficiente la calidad de la implementación del PMAP por parte del Servicio de Biodiversidad y Áreas Silvestres Protegidas (SBAP). En ese sentido, agregándose la aprobación del PMAP a las materias que requieren informe previo del artículo 48 bis de la Ley 19300 , de 1994, el Ministerio de Medio Ambiente (MMA) podría controlar preventivamente los estándares de calidad para un PMAP específico. Además, existiría cierta separación organizacional entre el Ministerio en su rol de "regulador", el Servicio en su rol de "prestador" y la Superintendencia en su rol de "fiscalizador", estando ésta última facultada para controlar al SBAP sólo en ese instrumento.

Como señala la OCDE, en Chile no hay reguladores independientes, sino meras agencias administrativas, pues, para ser designadas como reguladores independientes, su diseño y administración deben garantizar que estén orgánicamente libres de interferencia política contingente, que sean capaces de resistir la captura por grupos de interés sin dejar de ser responsivas, y que aseguren a todos los interesados debida transparencia y accesibilidad en su gestión. ${ }^{73}$ Debe también tenerse presente que la OCDE considera que en Chile la efectividad de la actividad regulatoria únicamente es monitoreada a través del cumplimiento de metas de gestión que son definidas y evaluadas por la misma organización que debe cumplirlas, y que no existe una organización externa e independiente que asegure que éstas efectivamente se cumplan, aunque exista el Consejo de Auditoría Interna General de Gobierno, pues éste monitorea para prevenir problemas de probidad administrativa y transparencia, de procesos de gestión de riesgos, y de control interno financiero y no financiero. ${ }^{74}$

${ }^{72}$ Elaboración propia a partir del Registro Nacional de Áreas Protegidas, http://rnap.mma.gob.cl/.

${ }^{73}$ OCDE, Reviews of Regulatory Reform, 69-70.

${ }^{74}$ Ibídem, 71. 
Por eso, coincidimos con la OCDE cuando recomienda a Chile que revise la estructura de gobernanza de sus reguladores, a través de modificaciones legales, para protegerla de cambios políticos e interferencia de la administración contingente, en particular cuando estos deben mantener la confianza pública o sus decisiones pueden tener impactos significativos que hacen imperativa su verdadera imparcialidad. Es preferible instalar diseños de gobernanza colegiada con periodos escalonados de ejercicio, involucrando en su designación y remoción anticipada al poder legislativo, y con normas estrictas y claras para manejar conflictos de intereses. ${ }^{75}$

Por tanto, coincidimos en el llamado de atención para que se modifiquen los mecanismos de designación y remoción de los directivos de la Superintendencia de Medio Ambiente, el Servicio de Evaluación Ambiental y el Servicio de Biodiversidad y Áreas Silvestres Protegidas, en particular que en su designación y remoción anticipada deba participar el poder legislativo. En cambio, respecto de los administradores de áreas protegidas, recomendamos establecer un sistema de control similar al propuesto en la reforma educativa respecto de los directores ejecutivos de cada servicio local de educación; y, en este caso, el administrador del área protegida debería ser designado por un período fijo, pudiendo ser removido del cargo anticipadamente por negligencia en el desempeño de sus funciones, entendiendo como tal los casos en que el administrador incurre en reiteración de infracciones graves al PMAP, así determinados en procedimiento sancionatorio de la SMA.

Como hemos expresado, una parte fundamental para el éxito de la política pública depende de la fijación de reglas formales, pues basadas en ellas se tendrán prácticas organizacionales, es decir, la manera real en que sus destinatarios, los individuos que componen las organizaciones, van a implementar y cumplir, total o parcialmente, con estas reglas.

$\mathrm{Si}$ bien las reglas formales no parecen contener incentivos o restricciones inherentes a la conducta organizacional, funcionaria o individual, es el contexto institucional el que une reglas formales e informales. La permanente vigilancia del cumplimiento de las primeras junto a la corrección de sus infracciones es lo que refuerza o refrena los incentivos o restricciones contenidos en el diseño institucional. Situar la fiscalización de los PMAP de las áreas protegidas estatales en la mis-

75 Ibídem, 77. 
ma organización que las administra contiene un incentivo a una labor complaciente en esa materia, afectando el propio objetivo del proyecto, que no es otro que gestionar adecuadamente la conservación de la diversidad biológica del país a través de los mecanismos de cumplimiento adecuados. En ese sentido, el proyecto de ley en discusión debería subsanar, antes de ser promulgado, estas brechas estructurales.

\section{BIBLIOGRAFÍA}

Baldwin, Robert, Martin Cave \& Martin Lodge. Understanding Regulation. Oxford: Oxford University Press, 2012.

Becker, Gary. "Crime and Punishment: An Economic Approach". Journal of Political Economy 76, n. ${ }^{\circ} 2$ (1968): 169-217.

Benavides, Paula, Rubén Castro \& Ingrid Jones. Sistema público de salud: Situación actual y proyecciones fiscales 2013-2050. Santiago: Dipres, 2013.

Boin, Arjen, Oliver James \& Martin Lodge. "The New Public Management 'Revolution' in Political Control of the Public Sector: Promises and Outcomes in Three European Prison Systems". Public Policy and Administration 21, n. ${ }^{\circ}$ 2 (2006): 81-100.

Brunsson, Nils \& Bengt Jacobsson. A World of Standards. Oxford: Oxford University Press, 2000.

Castro, Rodrigo, Lorenzo Cerda, Julio Neme \& Yael Senerman. "Evaluación y propuesta del sistema de control estratégico y operacional de Chile". En Un mejor Estado para Chile, editado por Visnja Tomicic \& Cristián García, 589612. Santiago: Andros Impresores, 2009.

Conama. Política Nacional de Áreas Protegidas. Santiago: Conama, 2005.

Crawford, Sue \& Elinor Ostrom. "A Grammar of Institutions". American Political Science Review 89, n. 3 (1995): 582-600.

Del Fávero, Gabriel. "Ley sobre bases generales del medio ambiente". Estudios Públicos 54 (1994): 1-46.

Den Heyer, Garth. "New Public Management: A Strategy for Democratic Police Reform in Transitioning and Developing Countries". Policing: An International Journal of Police Strategies \& Management 34, n. 3 (2011): 419-433.

Espinoza, Guillermo. Evaluación ambiental estratégica de apoyo al diseño del Sistema Nacional de Áreas Protegidas. Santiago: Andros, 2011.

Falabella, Alejandra. "El mercado escolar en Chile y el surgimiento de la nueva gestión pública”. Educação \& Sociedade 36, n. ${ }^{\circ} 132$ (2015): 699-722.

Figueroa, Verónica, Mauricio Olavarría y Bernardo Navarrete. "Política de modernización de la gestión pública en Chile 1990-2006”, Convergencia 18, n..$^{\circ} 57$ (2011): 61-99. 
Fuentes, Eduardo \& Rodolfo Domínguez. Aplicación y resultados de la Encuesta sobre Efectividad de Manejo de las principales áreas protegidas de Chile. Santiago: Andros, 2011.

Hodgson, Geoffrey. "What Are Institutions?" Journal of Economic Issues 40, n. ${ }^{\circ} 1$ (2006): 1-25.

Hood, Christopher. "A Public Management for all Seasons?" Public Administration 69 (1991): 3-19.

- The Blame Game: Spin, Bureaucracy, and Self-preservation in Government. Princeton: Princeton University Press, 2011.

Hood, Christopher, Oliver James, George Jones, Colin Scott \& Tony Travers. Regulation Inside Government: Waste-Watchers, Quality Police, and SleazeBusters. Oxford: Oxford University Press, 1999.

Huntington, Samuel. "Political Development and Political Decay". World Politics 17, n. ${ }^{\circ} 3$ (1965): 386-430.

Lodge, Martin \& Christopher Hood. "Regulation Inside Government: RetroTheory Vindicated or Outdated?". En The Oxford Handbook of Regulation, editado por Robert Baldwin, Martin Cave \& Martin Lodge, 590-609. Oxford: Oxford University Press, 2010.

Lodge, Martin \& Kai Wegrich. Managing Regulation: Regulatory Analysis, Politics and Policy. Londres: Palgrave Macmillan, 2012.

McFee, Graham. Sport, Rules and Values. Londres: Routledge, 2004.

Morales, Marjorie. "Nueva gestión pública en Chile: orígenes y efectos". Revista de Ciencia Política 34, n. ${ }^{\circ} 2$ (2014): 417-438.

North, Douglass. "Institutions and Economic Growth: A Historical Introduction". World Development 17 (1989): 1319-1332.

- Institutions, Institutional Change, and Economic Performance. Cambridge: Cambridge University Press, 1987.

OCDE. Environmental Performance Reviews: Chile 2016. París: OECD Publishing, 2016.

- Reviews of Regulatory Reform: Regulatory Policy in Chile. París: OECD Publishing, 2016.

—. Taking Stock of Regulatory Reform. Paris: OECD Publishing, 2005.

Ostrom, Elinor. "An Agenda for the Study of Institutions". Public Choice 48 (1986): 3-25.

Praus, Sergio, Mario Palma \& Rodolfo Domínguez. La situación jurídica de las actuales áreas protegidas de Chile. Santiago: Andros, 2011.

Simonet, Daniel. "The New Public Management Theory and European Health-care Reforms". Canadian Public Administration 54, n. ${ }^{\circ} 4$ (2008): 617-635.

Speidel, Gerhard. "Problemas de administración forestal en América Latina". Revista Bosque 1, n. ${ }^{\circ} 1$ (1975): 46-47.

Tolofari, Sowaribi. "New Public Management and Education". Policy Futures in Education 3, n. ${ }^{\circ} 1$ (2005): 75-89. EP 